\title{
Testing Lorentz Invariance Violation at the Pierre Auger Observatory
}

\author{
Rodrigo Guedes Lang ${ }^{* a}$ for the Pierre Auger Collaboration ${ }^{\dagger b}$ \\ ${ }^{a}$ Instituto de Física de São Carlos, Universidade de São Paulo, Avenida Trabalhador \\ São-Carlense 400, CEP 13566-590, São Carlos, SP, Brazil \\ ${ }^{b}$ Observatorio Pierre Auger, Av. San Martín Norte 304, 5613 Malargüe, Argentina \\ E-mail: auger_spokespersons@fnal.gov \\ Full author list: http://www.auger.org/archive/authors_icrc_2019.html
}

\begin{abstract}
In this work, we present the capabilities of testing Lorentz invariance violation (LIV) using data from the Pierre Auger Observatory. Two independent scenarios are treated. Firstly, LIV on the hadron sector is considered and the modified UHECR propagation obtained. An updated combined fit of the spectrum and composition data is performed for each LIV assumption. The data is better described with a low maximum rigidity at sources, which leads to a poor sensitivity to LIV effects. Secondly, subluminal LIV on the photon sector is considered and the propagation of GZK photons is modified. For one of the scenarios considered, very competitive limits on the LIV coefficient of the order of $\delta_{\gamma}^{(1)} \gtrsim-10^{-40} \mathrm{eV}^{-1}$ and $\delta_{\gamma}^{(2)} \gtrsim-10^{-60} \mathrm{eV}^{-2}$ are imposed. Finally, we discuss a promising possibility of testing LIV using the development of UHE air-showers.
\end{abstract}

36th International Cosmic Ray Conference - ICRC2019

24 July - 1 August, 2019

Madison, Wisconsin, USA

${ }^{*}$ Speaker.

${ }^{\dagger}$ for collaboration list see PoS(ICRC2019)1177 


\section{Introduction}

Relativity has been widely established and well tested during the last century. However, departures from relativity such as Lorentz invariance violation (LIV) have been proposed by several high energy models [1]. Potential LIV signatures are expected from several different astrophysical contexts. Previous studies have searched for LIV signals using distinct results, such as the time of flight of photons generated in gamma-ray bursts [2,3,4], modulations of the energy spectrum in TeV gamma-rays [5, 6, 7] and ultra-high energy cosmic rays (UHECR) [8, 9], and upper limits on the flux of EeV photons [10, 11]. No LIV signal has yet been found [1] and, thus, restrictive limits on the violation have been set [12].

LIV effects are expected to be suppressed at low energies and for short travel distances [1]. Consequently, UHECRs represent a unique possibility for testing it, being the most energetic known particles in the Universe [13] and coming from extragalactic sources [14].

The Pierre Auger Observatory is the largest experiment ever built to study UHECRs. It uses a hybrid technique composed of a surface detector (SD) with 1660 water-Cherenkov stations and a fluorescence detector (FD) with 27 fluorescence telescopes covering an area of $3000 \mathrm{~km}^{2}$. In this study, three important results obtained by the Observatory are used to search for LIV signals: (i) the energy spectrum of UHECR measured with unprecedented statistics [15], (ii) the distribution of depths of maximum of air-shower profiles, $X_{\max }$ [16], and (iii) the upper limits on the photon flux [17].

A generic phenomenological approach based on the Coleman \& Glashow formalism [18] is used here to introduce LIV as presented in section 2. Two different and independent LIV scenarios are tested: (a) LIV in the hadronic sector is tested by searching the best description of the energy spectrum and $X_{\max }$ distributions under LIV assumptions and (b) LIV in the photon sector is limited by the upper limits in the photon flux. A third promising scenario to search for LIV signals in the pion section by looking into the development of extensive air showers (EAS) is also discussed.

\section{Lorentz invariance violation framework}

The phenomenological approach of Coleman \& Glashow [18] summarizes LIV effects in a perturbative modification of the particle dispersion relation,

$$
E_{i}^{2}=p_{i}^{2}+m_{i}^{2}+\sum_{n=0}^{\infty} \delta_{i}^{(n)} E_{i}^{n+2}
$$

where $E, p$ and $m$ are, respectively, the energy, the momentum and the mass of the particle; $i$ denotes the particle species and $n$ the approximation order, while $\delta_{i}^{(n)}$ is the LIV coefficient for each particle at a given order. Searches for LIV use data to measure or limit values of $\delta_{i}^{(n)}$. It is usual to consider in each LIV analysis only one particle species and specific order, leaving $\delta_{i}^{(n)} \neq 0$ as a free parameter. Modifying the dispersion relation may lead to several effects depending on the particle considered and on the sign of $\delta_{i}^{(n)}$. In particular, the kinematics of some interactions are expected to be modified, resulting in a shift in their energy threshold.

In this work, two different and independent LIV scenarios are tested against Auger data: (a) positive LIV coefficients for the hadron sector $\left(\delta_{\text {had }}=\delta_{p}=\delta_{\pi}>0\right.$ ) and (b) negative LIV coeffi- 
cients for the photon sector $\left(\delta_{\gamma}<0\right)$. A third hypothesis is discussed for negative LIV coefficients for the pion sector $\left(\delta_{\pi}<0\right)$.

\section{UHECR propagation}

The spectrum of UHECRs propagating in the intergalactic medium is shaped by their interaction with the photon background. For the highest energies, the most relevant interactions are the photodisintegration for nuclei, ${ }^{A} Z+\gamma_{\mathrm{bkg}} \rightarrow{ }^{A^{\prime}} Z^{\prime}+p(n)$, and the photopion production for protons, $p+\gamma_{\mathrm{bkg}} \rightarrow p+\pi$. Changing the particle dispersion relation leads to a change in the kinematics of the interactions, causing modifications in the extragalactic propagation of the UHECRs. For this reason, LIV effects are expected to be visible in the measured spectrum and composition $[8,9]$ and, thus, these measurements can be used to search for LIV signatures.

In this section, LIV is considered in the hadron sector, $\delta_{p}=\delta_{\pi}=\delta_{\text {had }}>0$, and the modified kinematics of the photopion production and photodisintegration as published in [19] are used. The main effect of LIV is an increase in the mean free path of the interactions. The difference from the LI scenario becomes significant above a given energy for each LIV coefficient. The modified mean free paths are implemented in the SimProp code [20] and the resulting spectra and composition on Earth for each LIV assumption are obtained.

Identical and homogeneously distributed UHECR sources are considered emitting four different nuclear species $\left({ }^{1} \mathrm{H},{ }^{4} \mathrm{He},{ }^{14} \mathrm{~N},{ }^{28} \mathrm{Si}\right)$ with an energy spectrum given by a power law with a broken exponential cutoff,

$$
\frac{\mathrm{d} N_{A}}{\mathrm{~d} E}=J_{A}(E)=f_{A} J_{0}\left(\frac{E}{10^{18} \mathrm{eV}}\right)^{-\gamma} \times \begin{cases}1 & \text { for } E<Z_{A} R_{\text {cut }} \\ \exp \left(1-\frac{E}{Z_{A} R_{\text {cut }}}\right) & \text { otherwise }\end{cases}
$$

where $f_{A}$ is the fraction of the injected isotope over the total and is defined at fixed energy, $E=$ $10^{18} \mathrm{eV}, \gamma$ is the spectral index, $R_{\text {cut }}$ is the maximum rigidity at the sources.

The simulations took into account three LIV coefficients for the leading order $n=0, \delta_{\text {had }}{ }^{(0)}=$ $\left\{5 \times 10^{-24}, 10^{-23}, 10^{-22}\right\}$. The two limiting cases, LI $\left(\delta_{\text {had }}=0\right)$ and maximum LIV $\left(\delta_{\text {had }} \rightarrow \infty\right)$, were also considered. The maximum LIV scenario represents the propagation of UHECR with no interactions with the photon backgrounds at all energies, taking into account only the adiabatic energy losses due to the expansion of the universe.

The analysis searches for the values of the parameters which best describe the energy spectrum [15] and the $X_{\max }$ distributions [16] for each LIV assumption, updating the results reported in [9]. The fit procedure follows a method similar to the one described in [21]. Only energies above $E>10^{18.7} \mathrm{eV}$ are considered; the combined energy spectrum is employed and Gumbel parametrizations are used to fit the $X_{\max }$ distributions. The goodness of fit is obtained using a generalized $\chi^{2}$ (the deviance, $D=-2\left(\ln \left(L_{J} / L_{J}^{\text {sat }}\right)+\ln \left(L_{X_{\max }} / L_{X_{\max }}^{\text {sat }}\right)\right)$ ). EPOS-LHC [22] is used as hadronic model for interactions in the atmosphere.

Tab. 1 shows the results of the fits for each of the LIV assumptions. The results using the 2017 data set [15, 16] confirm the finding of [9], where the best fit found using the 2015 data set [23, 24] is located in an elongated region extended below $\gamma=1$. The value of the deviance is worse with respect to the one reported in [9], both for the LIV and the LI cases, due to the increased statistics 


\begin{tabular}{l|cc|cccc|cc|c}
\hline Scenario & $\gamma$ & $\lg \left(R_{\text {cut }} / V\right)$ & $f_{\mathrm{H}}$ & $f_{\mathrm{He}}$ & $f_{\mathrm{N}}$ & $f_{\mathrm{Si}}$ & $D(J)$ & $D\left(X_{\max }\right)$ & $D_{\text {total }}$ \\
\hline LI, $\delta_{\text {had }}=0$ & -1.13 & 18.25 & 70.1 & 29.5 & 0.4 & 0.02 & 19.9 & 236.6 & 256.5 \\
\hline LIV, $\delta_{\text {had }}{ }^{(0)}=5 \times 10^{-24}$ & -1.26 & 18.24 & 68.9 & 30.8 & 0.3 & 0.02 & 19.5 & 235.6 & 255.1 \\
LIV, $\delta_{\text {had }}{ }^{(0)}=1 \times 10^{-23}$ & -1.20 & 18.25 & 67.4 & 32.2 & 0.4 & 0.02 & 19.9 & 236.1 & 256.0 \\
LIV, $\delta_{\text {had }}{ }^{(0)}=1 \times 10^{-22}$ & -1.42 & 18.22 & 68.4 & 31.4 & 0.2 & 0.01 & 17.7 & 231.8 & 249.5 \\
\hline $\max$ LIV, $\delta_{\text {had }} \rightarrow \infty$ & 0.91 & 18.47 & 52.3 & 42.3 & 5.4 & 0. & 34.4 & 189.7 & 224.1 \\
\hline
\end{tabular}

Table 1: Best fit parameters for the LI reference model and LIV cases (using SimProp simulations).

of the data set. A better separation between the values of the minimum with respect to what is shown in Fig. 1 can be obtained for example using a different hadronic model in the atmosphere.

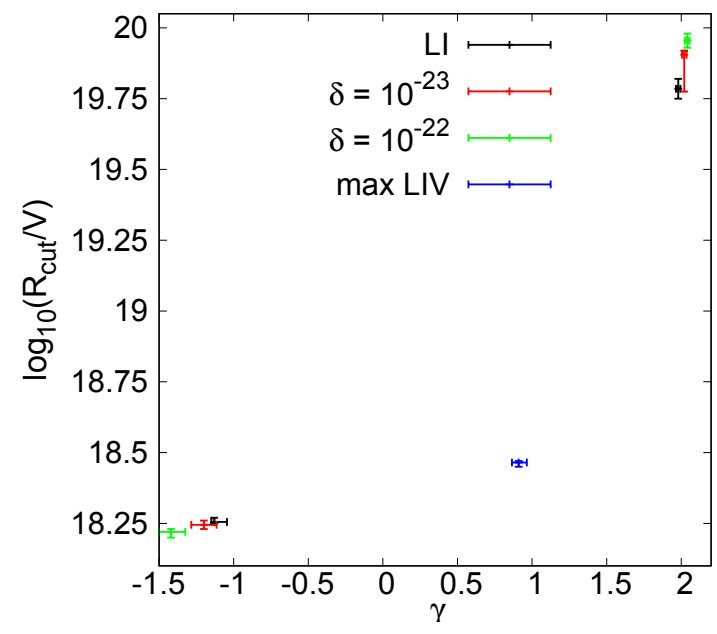

Figure 1: Position of the best-fit values as a function of the spectral index and rigidity cutoff, for the LI case and some of the LIV cases, are reported in the lower-left part of the figure. The error bars are computed from the interval $D \leq D_{\text {min }}+1$. The local minima are reported in the upper-right part of the plot: in this case the error bars are computed from the interval $D \leq D_{\text {local }}+1$.

\section{GZK photons propagation}

Propagating UHECRs interact with the background photons, emitting pions via photopion production. Neutral pions rapidly decay into EeV photons, called GZK photons, which may arrive on Earth. The Pierre Auger Observatory has the capability of detecting these photons. Nevertheless, no event has yet been detected and, consequently, restrictive upper limits on the photon flux have been imposed [17].

Propagating EeV photons interact with the background radiation via pair production, $\gamma+\gamma_{\text {bkg }}$ $\rightarrow e^{+}+e^{-}$, which strongly suppresses the expected flux of GZK photons [25]. In this section, we consider subluminal LIV in the photon sector for the leading orders $n=1,2\left(\delta_{\gamma}<0\right)$. The kinematics of the pair production considering LIV is obtained following the procedure described in Ref. [11]. The main effect in the propagation is an increase in the mean free path when LIV is considered, which leads to photons traveling farther and, consequently, to an enhanced flux of GZK photons.

Since the GZK photons are emitted during the propagation of UHECR, the assumptions about the UHECR sources, such as the injection energy, composition and redshift evolution, play an important role in the expected flux of GZK photons [11]. In this work, two astrophysical scenarios 


\begin{tabular}{l|cc|rrrrc}
\hline Scenario & $\gamma$ & $\lg \left(R_{\text {cut }} / V\right)$ & \multicolumn{1}{c}{$f_{\mathrm{H}}$} & \multicolumn{1}{c}{$f_{\mathrm{He}}$} & \multicolumn{1}{c}{$f_{\mathrm{N}}$} & \multicolumn{1}{c}{$f_{\mathrm{Si}}$} & $f_{\mathrm{Fe}}$ \\
\hline A & -1.5 & 18.185 & 60.00 & 39.70 & 0.20 & 0.02 & 0.00 \\
B & 1.97 & 19.86 & 0.01 & 0.00 & 47.10 & 52.90 & 0.00 \\
\hline
\end{tabular}

Table 2: Best fit parameters (corresponding to the the global, A, and local, B, minima of the combined fit) for the LI model obtained with CRPropa simulations.
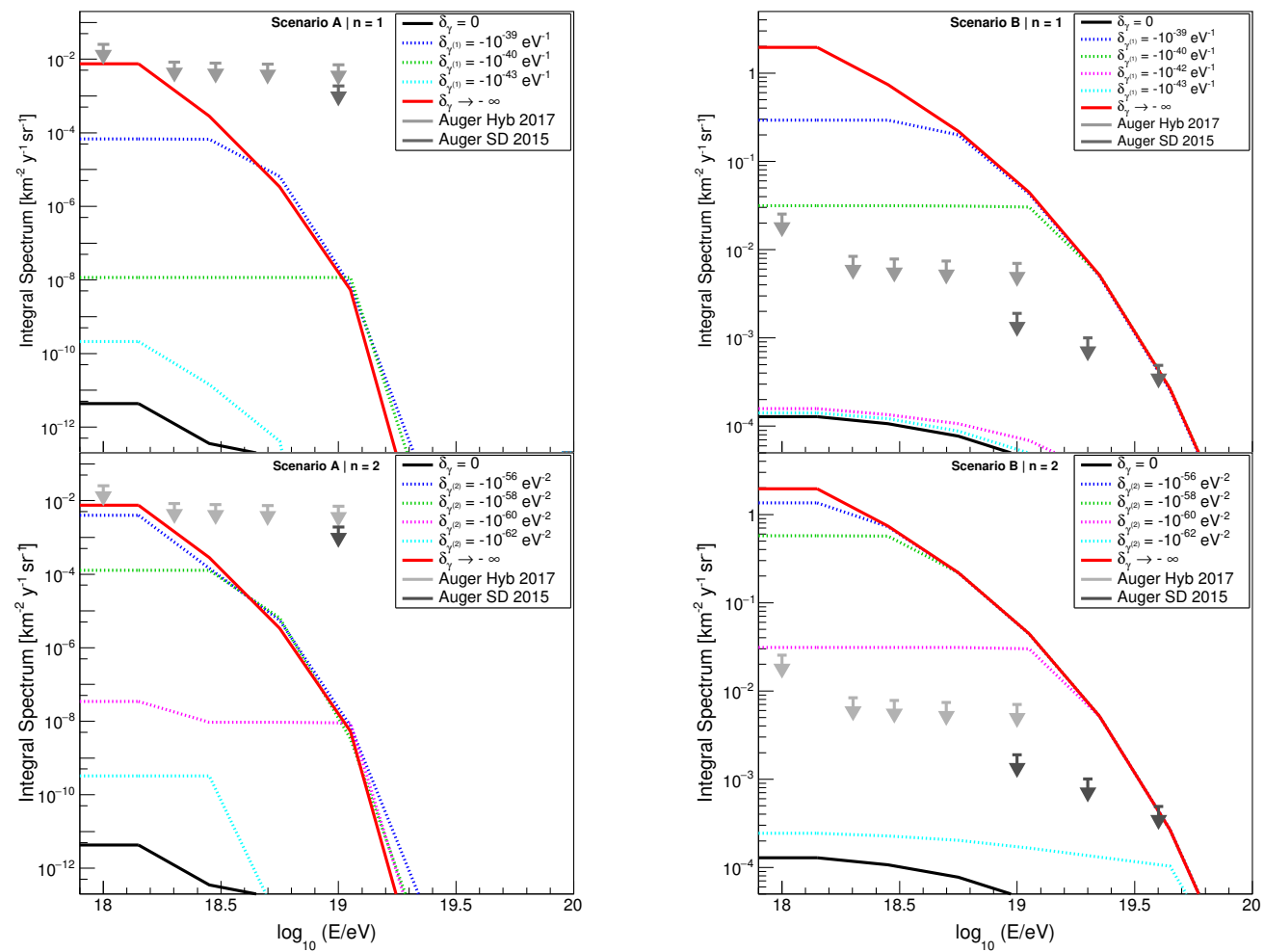

Figure 2: Integral flux of GZK photons. The left and right panels represents scenarios A and B, respectively. Top and bottom panels represents LIV approximation orders $n=1$ and $n=2$, respectively. The continuous black line represents the LI scenario, the continuous red line represents the maximum LIV scenario and the dashed colored lines represent intermediate LIV scenarios. Gray and black arrows show the upper limits from the Pierre Auger Observatory [17].

are considered. These scenarios come from the combined fit described in the previous section, using a LI propagation simulated with CRPropa3 [26]. The first one, A, is the best fit, with hard spectral index, while the second one, $\mathrm{B}$, is a local minimum, corresponding to a softer spectral index (see Tab. 2). Both scenarios consider identical and homogeneous sources distributed up to $9600 \mathrm{Mpc}$.

The LIV photon mean free paths were implemented in CRPropa3/EleCa [26] and several intermediate LIV scenarios are considered for the leading orders $n=1,2$. The two limiting cases are also considered, i.e., $\operatorname{LI}\left(\delta_{\gamma}=0\right)$ and maximum $\operatorname{LIV}\left(\delta_{\gamma} \rightarrow-\infty\right)$.

Fig. 2 shows the resulting integral fluxes of GZK photons compared to the upper limits on the flux from the Pierre Auger Observatory. As expected, when LIV is considered, the flux is stronger. 

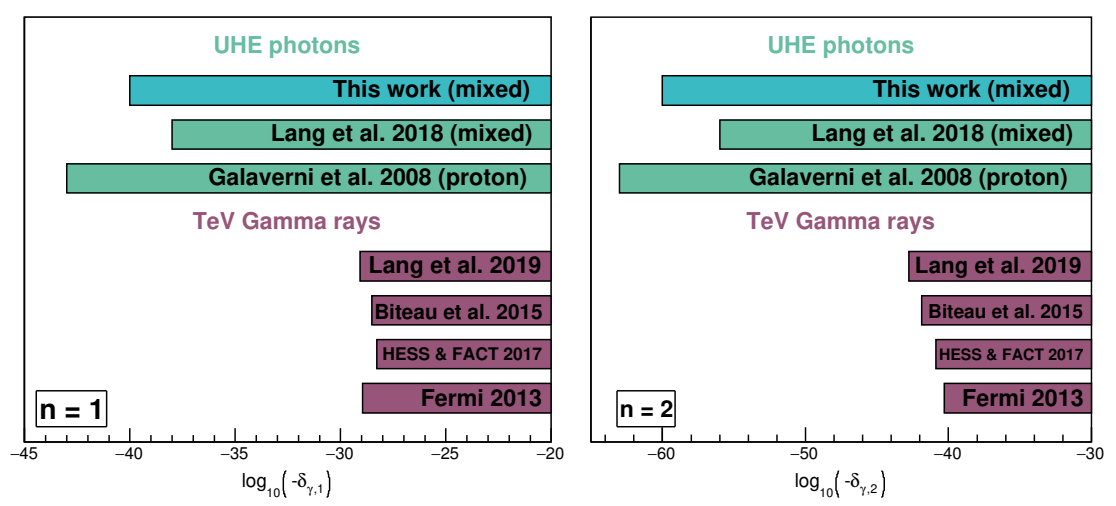

Figure 3: Limits on the subluminal LIV coefficient. The left and right panels show the limits for $n=1$ and $n=2$, respectively. The green bars show limits coming from UHE photons $[10,11]$ and the purple bars show limits coming from TeV gamma-rays [7, 5, 6, 2].

For the astrophysical scenario $A$, not even in the most extreme case, $\delta_{\gamma} \rightarrow-\infty$, the flux becomes larger than the upper limits and, thus, no LIV limits can be imposed. For the astrophysical scenario $\mathrm{B}$, on the other hand, some of the LIV coefficients considered are enough to create a flux larger than the upper limits and, consequently, limits on the LIV coefficient can be imposed. For this scenario, the imposed limits are $\delta_{\gamma}^{(1)} \gtrsim-10^{-40} \mathrm{eV}^{-1}$ and $\delta_{\gamma}^{(2)} \gtrsim-10^{-60} \mathrm{eV}^{-2}$.

Fig. 3 shows a comparison between the limits on the LIV coefficient for the subluminal photon sector imposed in this work and previous limits imposed from astrophysical tests. The comparison with limits from the $\mathrm{TeV}$ gamma-rays are not straight-forward since different astrophysical assumptions, photon energies and systematic uncertainties are considered in each case. Limits from refs. $[10,11]$ were imposed using a similar approach. Galaverni et al. considers pure proton composition at the source and the upper limits of the photon fluxes published in 2007 and 2008. A pure proton composition, however, is not favored by the $X_{\max }$ data [16]. Lang et al. [11] updated the analysis using mixed composition and the upper limits of the photon fluxes published in 2017. The limits imposed here are directly derived from the latest Pierre Auger data and are 2 and 4 orders of magnitude more restrictive than those imposed Lang et al. [11] for $n=1$ and $n=2$ respectively.

\section{Extensive air showers development}

One of the key interactions which governs the EAS development is the neutral pion decay, $\pi^{0} \rightarrow \gamma+\gamma$, because it connects the hadronic and electromagnetic components of the shower. If LIV is considered in the pion sector, the kinematics of the pion decay are affected and thus the shower development.

Considering the total energy of the pion, $s_{i}^{2}=E_{\pi}^{2}-p_{\pi}^{2}=m_{\pi}^{2}+\sum \delta_{\pi}^{(n)} E_{\pi}^{n+2}=s_{f}^{2}$, one can show that for certain values $\delta_{\pi}<0$, the decay becomes kinematically forbidden above $E_{\pi}^{(n)}>m_{\pi}^{2} / \delta_{\pi, n}$.

In this work, the changes in the pion decay due to possible LIV were implemented in the CONEX [27] software. In particular, a LIV coefficient of $\delta_{\pi}=-1 / M_{\mathrm{Pl}} \approx-8.2 \times 10^{-29} \mathrm{eV}^{-1}$ has been considered. About 5000 air-showers were simulated for each mass (hydrogen, helium, nitrogen, iron) and each energy bin in the range from $10^{17}$ up to $10^{20} \mathrm{eV}$ in logarithmic steps of 0.25 . In 

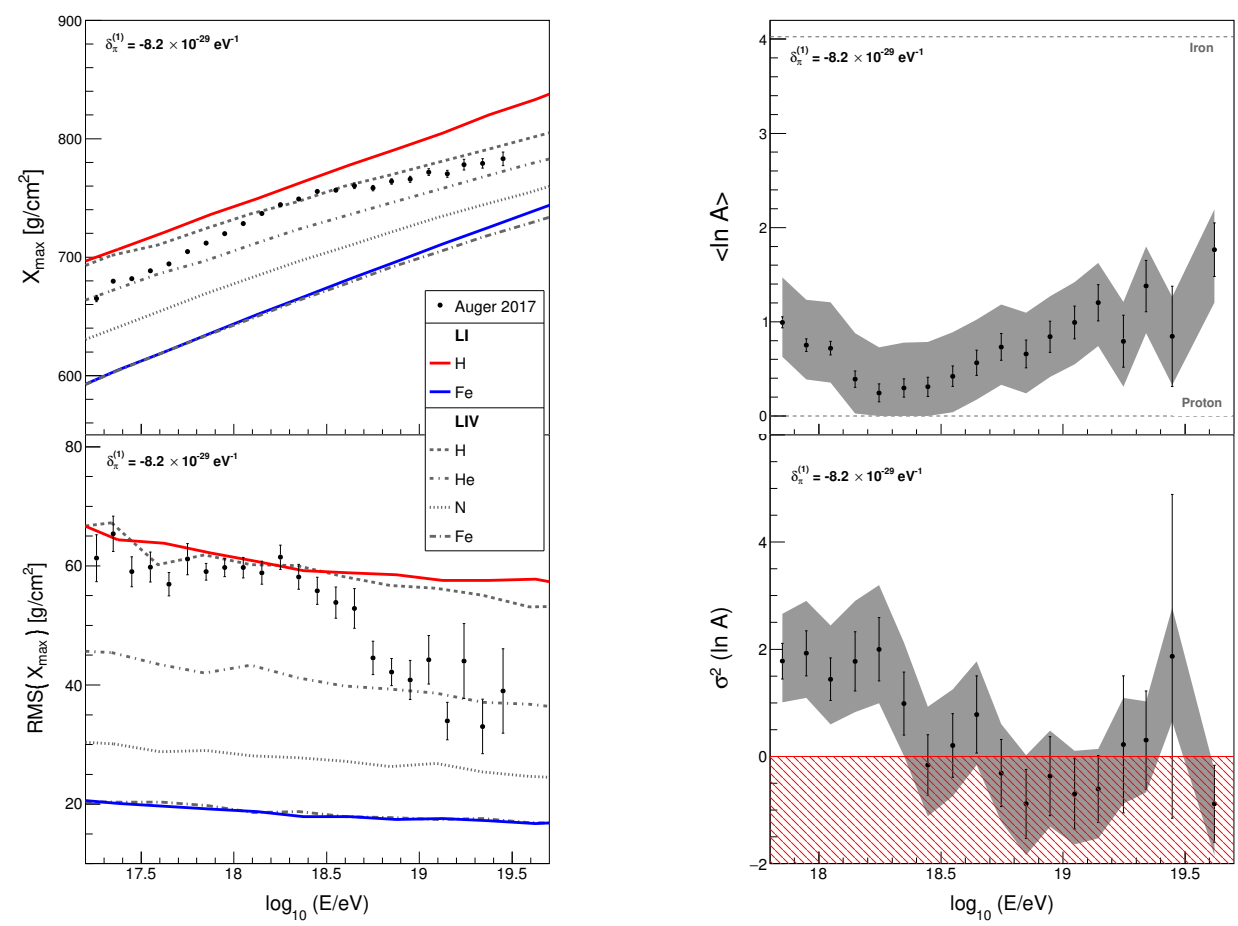

Figure 4: Left panel: First two moments of the $X_{\max }$ distribution. The red and blue continuous lines show the simulated moments for a pure composition of proton and iron, respectively, in a LI scenario. The dashed lines show the simulated moments for a LIV scenario with $\delta_{\pi}^{(1)}=-8.2 \times 10^{-29} \mathrm{eV}^{-1}$. The black points show data measured by the Pierre Auger Observatory [16]. Right panel: First two moments of the $\ln A$ distribution for a LIV scenario with $\delta_{\pi}^{(1)}=-8.2 \times 10^{-29} \mathrm{eV}^{-1}$. The black dots represent the simulated data and the gray area represents the systematic uncertainties of the Observatory.

Fig. 4, on the left panel, the first and second moments of the $X_{\max }$ distributions for the LI and LIV scenarios are shown. As it can be noted, the $\left\langle X_{\max }\right\rangle$ is sensitive to LIV and the composition behaves as if it were lighter in the LIV scenario. On the right panel, the resulting $\langle\ln A\rangle$ and $\sigma^{2}(\ln A)$ obtained from the first two moments, using the modified hadronic interaction model EPOS-LHC, are shown. It is straightforward to notice that, in presence of LIV, the $\sigma^{2}(\ln A)$ ends up into the the nonphysical region below zero. This behaviour is more pronounced in the LIV scenario with respect to what has been found in the LI case with the same hadronic interaction model $[28,16]$.

\section{Conclusions}

This paper tests Lorentz invariance violation using the Pierre Auger Observatory data.

First, an update of our previous work [9] was presented. LIV was considered in the propagation of UHECRs by modifying the photopion production and photodisintegration. The LIV simulations were fitted to updated data of the spectrum and $X_{\max }$ distribution. The data is better described by a scenario with relatively low maximum rigidity, reducing the potentiality of the measurements to reveal LIV effects, which are suppressed by the energy.

In the second scenario, the procedure proposed in Ref. [11] is followed for GZK photons. Considering a scenario in which the UHECR spectrum is described by a model with high rigidity 
at the sources, limits of the order of $\delta_{\gamma}^{(1)} \gtrsim-10^{-40} \mathrm{eV}^{-1}$ and $\delta_{\gamma}^{(2)} \gtrsim-10^{-60} \mathrm{eV}^{-2}$ are imposed. These limits are 2 and 4 orders of magnitude more restrictive than the most updated ones using the same technique [11] for $n=1$ and $n=2$, respectively.

Finally, we discuss the possibility of using extensive air shower to test LIV. The pion decay is changed and promising preliminary results show that its effects on the $X_{\max }$ distribution can be used in the future to impose limits on LIV.

\section{Acknowledgements}

We acknowledge the support from Fundação de Amparo à Pesquisa do Estado de São Paulo (FAPESP) through grants 2015/15897-1, 2016/24943-0 and 2019/01653-4. We also acknowledge the National Laboratory for Scientific Computing (LNCC/MCTI, Brazil) for providing HPC resources of the SDumont supercomputer, which have contributed to the research results reported within this paper (sdumont.lncc.br).

\section{References}

[1] D. Mattingly, Living Rev. Rel. 8, 5 (2005) doi:10.12942/lrr-2005-5 [gr-qc/0502097].

[2] V. Vasileiou et al., Phys. Rev. D 87, 122001 (2013) [arXiv:1305.3463].

[3] J. Ellis et al., Phys. Rev. D 99, 083009 (2019) [arXiv:1807.00189].

[4] H. Abdalla and M. Böttcher, Astrophys. J. 865, 159 (2018) [arXiv:1809.00477].

[5] J. Biteau and D.A. Williams, Astrophys. J. 812, 60 (2015) [arXiv:1502.04166].

[6] G. Cologna et al. [H.E.S.S. and FACT Collaborations], AIP Conf. Proc. 1792, 050019 (2017) [arXiv:1611.03983].

[7] R.G. Lang et al., Phys. Rev. D 99, 043015 (2019) [arXiv:1810.13215].

[8] D. Boncioli et al., PoS ICRC 2015, 521 (2016) [arXiv:1509.01046].

[9] D. Boncioli [Pierre Auger Collaboration], PoS ICRC 2017, 561 (2018).

[10] M. Galaverni and G. Sigl, Phys. Rev. Lett. 100, 021102 (2008) [arXiv:0708.1737].

[11] R. Guedes Lang et al., Astrophys. J. 853, 23 (2018) [arXiv:1701.04865].

[12] S. Liberati and L. Maccione, Ann. Rev. Nucl. Part. Sci. 59, 245 (2009) [arXiv:0906.0681].

[13] A. Aab et al. [Pierre Auger Collaboration], JCAP 1508, 049 (2015) [arXiv:1503.07786].

[14] A. Aab et al. [Pierre Auger Collaboration], Science 357, no. 6537, 1266 (2017) [arXiv:1709.07321].

[15] F. Fenu [Pierre Auger Collaboration], PoS ICRC 2017, 486 (2018).

[16] J. Bellido [Pierre Auger Collaboration], PoS ICRC 2017, 506 (2018).

[17] A. Aab et al. [Pierre Auger Collaboration], JCAP 1704, 009 (2017) [arXiv:1612.01517].

[18] S.R. Coleman and S.L. Glashow, Phys. Rev. D 59, 116008 (1999) [hep-ph/9812418].

[19] R.G. Lang, Master Thesis, University of São Paulo, Brazil (2017).

[20] R. Aloisio et al., JCAP 1711, 009 (2017) [arXiv:1705.03729].

[21] A. Aab et al. [Pierre Auger Collaboration], JCAP 1704, 038 (2017), Erratum: [JCAP 1803, E02 (2018)] [arXiv:1612.07155].

[22] T. Pierog et al., Phys. Rev. C 92, 034906 (2015) [arXiv:1306.0121].

[23] I. Valino [Pierre Auger Collaboration], PoS ICRC 2015, 271 (2016).

[24] A. Porcelli [Pierre Auger Collaboration], PoS ICRC 2015, 420 (2016).

[25] A. De Angelis et al., Mon. Not. Roy. Astron. Soc. 432, 3245 (2013) [arXiv:1302.6460].

[26] R. Alves Batista et al., JCAP 1605, 038 (2016) [arXiv:1603.07142].

[27] T. Bergmann et al., Astropart. Phys. 26, 420 (2007) [astro-ph/0606564].

[28] P. Abreu et al. [Pierre Auger Collaboration], JCAP 02, 026 (2013). 